\title{
Variable sonographic spectrum of parathyroid adenoma with a novel ultrasound finding: dual concentric echo sign
}

\author{
Turker Acar ${ }^{1}$, Suha Sureyya Ozbek², Yesim Ertan ${ }^{3}$, Gulgun Kavukcu², Muge Tuncyurek ${ }^{3}$, \\ Recep Gokhan Icoz ${ }^{4}$, Mehmet Mahir Akyildiz ${ }^{4}$, Ozer Makay, Seval Acar ${ }^{5}$
}

${ }^{1}$ Department of Radiology Abant Izzet Baysal University School of Medicine Izzet Baysal Education and Training Hospital, Bolu, ${ }^{2}$ Department of Radiology, Ege University School of Medicine, Izmir, ${ }^{3}$ Department of Pathology, Ege University School of Medicine, Izmir, ${ }^{4}$ Department of Surgery, Ege University School of Medicine, Izmir, Turkey, ${ }^{5}$ Department of Family Medicine, Tepecik Education and Training Hospital, Izmir, Turkey

\begin{abstract}
Aims: To review the detailed gray-scale and Doppler ultrasonography features of histologically proven parathyroid adenomas (PAs) evaluated with high-end ultrasonography devices and to present a novel ultrasonography finding called the dual concentric echo sign in PA with histopathologic correlation which was encountered during detailed analysis. Material and methods: Fifty-six PAs with histopathological result were enrolled. The longest dimension, shape, distance to skin surface, internal echo and Doppler US features obtained with high-end US devices were evaluated. Results: PAs had variable range of shape including oval, irregular, fusiform, lobulated, crescent-shaped, and nodular configuration. In nine patients the lesions were shown to have cystic components and calcifications were seen in four cases. Dual concentric echo sign was detected in $18 \%$ PAs. Histological reevaluation of this subgroup demonstrated significantly increased edema $(\mathrm{p}<0.01)$, and ectatic vessels $(\mathrm{p}=0.02)$ in the central part of the lesion compared to the rest of the PAs. Conclusions: The results of the study led to the conclusion that PAs have variable gray-scale and Doppler findings. Typical sonographic features like ovoid shape, homogeneously hypoechoic pattern may not be present in all PAs. Dual concentric echo sign which is a novel sonographic pattern may be suggestive of a PA.
\end{abstract}

Keywords: Parathyroid adenoma, gray-scale ultrasound, Doppler ultrasonography, dual concentric echo sign.

\section{Introduction}

Excessive production of parathyroid hormone (PTH) is defined as hyperparathyroidism and classified in three groups; primary, secondary, and tertiary. Primary hyperparathyroidism, originating from autonomous hypersecretion of PTH, usually occurs in the setting of a parathyroid adenoma (PA) in $80 \%$ of patients, but can also be seen in parathyroid gland hyperplasia $(15-20 \%)$ or carcinoma $(0.5 \%)[1,2]$.

Received 10.01.2015 Accepted 28.02.2015

Med Ultrason

2015, Vol. 17, No 2, 139-146

Corresponding author: Turker Acar

Sağllk Mh. Şehitler Cad. 14300 Bolu, Turkey

Phone: : +90 (374) 2704575

Fax: +90 (374) 2534615

E-mail: drtacar@hotmail.com
Combined use of ultrasonography (US), PTH evaluation, and fine needle aspiration biopsy have $100 \%$ specificity and $90 \%$ sensitivity in diagnosing adenomatous disease in parathyroid glands [3]. Over the last decade, minimally invasive parathyroidectomy (MIP) has become the operation of choice for most patients with sporadic primary hyperparathyroidism. Using preoperative imaging as a tool to guide the operation, MIP has many advantages over the traditional four-gland exploration technique [4-6].

A typical PA appears uniformly hypoechoic in US examination. It has dimensions with at least two to three times the size of a normal gland giving a tear-drop appearance [7]. The capsule is usually distinct and hyperechoic relative to parenchyma [8]. On the other hand, larger adenomas may have lobulated configuration; they may contain cystic and/or calcified components. Occasionally, they may display increased internal echogenic- 
ity [9]. Classical hypoechoic, tear-drop shaped appearance, and typical blunt termination of a single vessel into the adenoma parenchyma which gives a "vascular arc" shape in Doppler imaging [10] enable an easy diagnosis in many US examinations. However, not all parathyoid adenomas may follow this rule.

In this study, we reviewed and correlated the preoperative sonoanatomic variations and histopathologic findings of PAs, which were ultrasonographically diagnosed or suspected in our center during the last ten years, and also, defined a novel US sign with histopathological correlation.

\section{Material and methods}

\section{Patients:}

The clinical, US, and histopathological data of 55 patients with 56 PAs (two PAs in the same patient) which had been ultrasonographically diagnosed or suspected, and later histopathologicaly proven in our hospital in the last 10 years were retrospectively analyzed. The demographic data, levels of serum calcium, intact PTH and urinary calcium excretion of the patients were retrieved from the digital hospital archive. Intact PTH measurements were performed with Beckman Coulter UniCel DxI 800 system chemiluminescence hormone analyzer (Beckman Coulter, Fullerton, CA, USA). The Institutional Ethics Committee approved this retrospective study and waived the informed consent requirement.

\section{US examinations:}

All gray-scale and Doppler US examinations were performed by the same radiologist experienced in this modality for more than 10 years and blinded to the laboratory and pathological results. During US examinations, high-resolution color Doppler US devices (Siemens Sonoline Antares and Siemens Acuson Antares scanners; Mountain View, CA, USA) equipped with multiple (1.5-dimensional) linear-array transducers (VFX9-4 and VFX13-5) were used. The frequency band-widths of these transducers ranged between 4-9 and 5-13 Megahertz $(\mathrm{MHz})$, respectively. Patients with suspicious clinical features of hyperparathyroidism had been routinely examined in supine position with slightly extended neck posture. In addition to the close neighborhood of the thyroid gland, retrotracheal area, suprasternal fossa, cervical regions adjacent to common carotid arteries, and also the upper neck including the submandibular compartment had been scanned for possible ectopic parathyroid lesions. All images were taken and stored using digital software.

Detailed US features of each PA including its location (relative to the thyroid gland), depth of its most superfi- cial edge from the skin surface, longest diameter (A) and the diameter (B) perpendicular to A in the same plane, shape (in the aforementioned plane), surface regularity, internal echogenicity, echo texture, Doppler sonographic features, in addition to the data regarding the presence of any cystic component or calcification were retrospectively reviewed and analyzed using the digital image and report archive. The location of PAs were assumed to be inferior (i.e., originating from the inferior parathyroid glands) when they were demonstrated in the close proximity to the lower poles of the thyroid gland. In accordance with the related literature, the lesions depicted behind the upper half or middle parts of each thyroideal lobe were accepted to have originated from the superior parathyroid glands [11]. Regarding the shape of PAs, completely circular lesions were accepted as nodular. On the other hand, ovoid PAs with an $\mathrm{A} / \mathrm{B}$ ratio less than 5.0 were assumed as oval, while the ones with an $\mathrm{A} / \mathrm{B}$ ratio equal or greater than 5.0 were considered to have a fusiform shape. Additionally, lobulated and crescentshaped PAs were named after their special configurations. Any PA with an amorphous appearance other than aforementioned forms was accepted to have an irregular shape. Surface regularity of PAs was classified as smooth or irregular. Any adenoma surface with a focal indentation deeper than $0.5 \mathrm{~mm}$ was accepted as irregular. The echo texture of a PA was accepted as homogeneous, when uniformly fine echoes were demonstrated within the lesion. Lesions with discrete internal echoes other than the dominant texture like septa, cysts, calcification distorting its homogeneity, were classified as heterogeneous. A cystic component was defined as any anechoic and well-bordered area with or without posterior acoustic enhancement, while any hyperechogenic focus with or without acoustic shadow was accepted as calcification. The internal echogenicity of PAs was assessed as hypo-, iso- or hyperechoic in comparison to that of thyroid gland. In some PAs dual concentric echo layers, each covering more than $1 / 4$ of the cross-sectional scan area were observed. In these cases, the echogenicity of the larger and dominant layer was selected to represent the echogenicity of the lesion. The cross-sectional color Doppler images of the PAs in the plane of their longest diameter were evaluated qualitatively to assign the vascularity of the lesion. In the case of color pixels covering less than approximately $10 \%$ of the PA, the lesion was decided to be hypovascular. Lesions with color pixels covering $10-50 \%$ or more than $50 \%$ of the cross-sectional area were accepted to be moderately or highly vascular, respectively. As described earlier, the demonstration of any vessel enveloping 90 to 270 degrees of the PA was accepted as a positive "vascular arc sign" [3]. 


\section{Surgical technique:}

The diagnosis of primary hyperparathyroidism was based on the presence of elevated serum calcium and PTH levels in the absence of kidney disease. All patients underwent an US and a sestamibi scan as preoperative localization tests. On the basis of the results of these tests, a selective (focused/targeted) parathyroidectomy or conventional unilateral/bilateral exploration was performed [12]. The procedures were carried out using a technique previously described by Miccoli et al [13]. Accordingly, operations were performed under local or general anesthesia, through a 2 to $4-\mathrm{cm}$ transverse neck incision, with or without the use of video assistance. Intraoperative PTH levels were determined as described elsewhere, and a decrease of circulating PTH level by $50 \%, 20$ minutes after excision of the enlarged parathyroid gland(s) was considered indicative of successful parathyroidectomy [14]. Most patients were discharged home the next day of surgery.

\section{Histopathological examinations:}

The pathologic materials of 56 PAs were assessed by the same histopathologist experienced in endocrine pathology. The basic criteria to establish adenoma diagnosis included a pushing border with an absence of intralesional fibroadipose tissue, a cell population demarcated from "normal" parathyroid tissue and absence of lobular growth [15]. Cell compositions of each adenomas were categorized as chief cell, oxphilic, and water-clear adenoma. Because some adenomas contained different cell combinations (i.e. chief and oxphilic cell; chief and water-clear cell), they were expressed as a mixed type depending on the most common two cell components. The histopathological findings like the longest diameter of PAs, presence /absence of normal parathyroid tissue around adenomatous tissue were compared to sonographic data of identical lesions. During the preparation of manuscript, the pathological materials of 54 lesions that could be achieved from pathology archive were reevaluated in terms of features such as the presence /absence of ectatic vessels, edema and fibrous bands within the PAs.

\section{Statistical analysis:}

The Kolmogorov Smirnov test was used to determine distribution of variables. Normally distributed data were expressed as mean \pm standard deviation (SD), whereas median, minimum-maximum and range values were used for numerical data which did not conform to normal distribution. The analyses between two means were performed with parametric Student's t test and non-parametric Mann-Whitney U test. $\chi^{2}$ test was used for comparison of ratios. The level of the significance was taken as $p$ $<0.05$. Statistical analyses were performed with SPSS ${ }^{\circledR}$ for Windows software (version 16.00, Chicago, IL).

\section{Results}

\section{Demographic features:}

Eight male and 47 female patients histopathologically diagnosed to have PA constituted the study group (mean age $\pm \mathrm{SD}, 51.5 \pm 13.5$ ). The mean age $\pm \mathrm{SD}$ of male and females were $63.3 \pm 15.7$ and $49.5 \pm \mathbf{1 2} .1$ years, respectively. One male patient had two PAs, each located caudal to lower poles of thyroideal lobes. Thus, the total number of PAs reached to 56 for PA-based analyses.

\section{Biochemical results:}

The serum calcium levels of 55 patients were found to be between 10.1 and $17.0 \mathrm{mg} / \mathrm{dL}$ (median: 11.3, minimum-maximum: 10.1-17, range: 6.9; reference values: 8.6-10.2). Intact serum parathormone levels in 48 patients were found to be between 74 and $2243 \mathrm{pg} / \mathrm{mL}$ (median: 123, minimum-maximum: 70-2243, range: 2173; reference values: 15-65). Urinary calcium excretion data was available for 37 patients in the retrospective analysis, and were found to be between 165 and $1385 \mathrm{mg}$ /day (median: 436, minimum-maximum: 165-1385, range: 1220 ; reference values: 100-300).

\section{Ultrasonographic features:}

The longest diameters of PAs ranged from 0.5 to 4.2 $\mathrm{cm}$, having a mean value of $1.89 \mathrm{~cm}( \pm 0.9 \mathrm{SD})$. The distance from the skin surface to the most superficial part of the PAs was $1.4 \mathrm{~cm}$ in average (SD: $0.4 \mathrm{~cm}$; range: $0.7-2.7 \mathrm{~cm}$ ). Other US parameters and the results of the analysis were depicted on Table I. PAs were found to have originated from inferior parathyroid glands in $80 \%$ of lesions. In two cases $(3.5 \%)$ ectopic adenomas were sonographically demonstrated in the right half of suprasternal fossa, where they lay deeply, adjacent to the bifurcation of brachiocephalic artery. One of them was located cranial, while the other was observed caudal to the bifurcation.

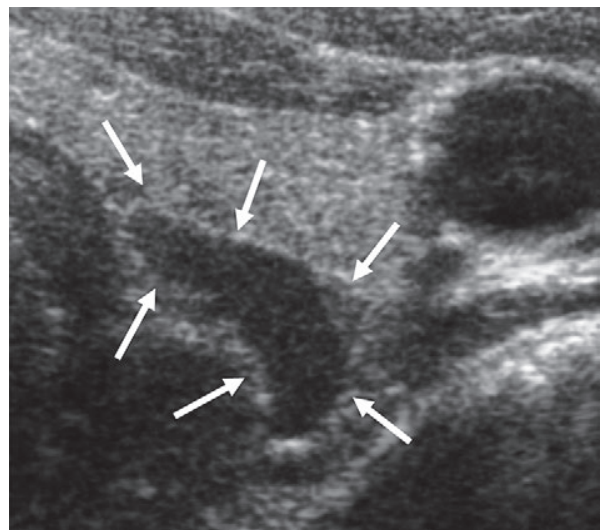

Fig 1. Transverse grey scale US image in a 57 year-old woman is depicted. There is a crescent shaped parathyroid adenoma adjacent to the left inferior half of thyroid gland (arrows). 


\begin{tabular}{lll} 
Table I. Ultrasonographic features of parathyroid adenomas. \\
\hline Sonographic Parameter & US Finding & $\begin{array}{l}\text { Number } \\
\mathbf{( \% )}\end{array}$ \\
\hline Location and glandular origin & Right superior & $2(3.5)$ \\
& Left superior & $7(12.5)$ \\
& Right inferior & $21(37.5)$ \\
& Left inferior & $24(42.8)$ \\
& Ectopic & $2(3.5)$ \\
Shape & Oval & $34(60.7)$ \\
& Irregular & $15(26.7)$ \\
& Fusiform & $4(7.1)$ \\
& Lobulated & $1(1.7)$ \\
& Crescent-shaped & $1(1.7)$ \\
& Nodular & $1(1.7)$ \\
Surface regularity & Smooth & $45(80)$ \\
Echo texture & Irregular & $11(20)$ \\
Echogenicity (Dominant) & Homogeneous & $28(50)$ \\
& Heterogeneous & $28(50)$ \\
& Hypoechoic & $51(91)$ \\
& Isoechoic & $4(7)$ \\
Vascularization & Hyperechoic & $1(2)$ \\
Dual-concentric echo sign & Absent & $46(82)$ \\
& Present & $10(18)$ \\
Cystic component & Absent & $47(84)$ \\
Calcification & Present & $9(16)$ \\
& Absent & $52(93)$ \\
& Present & $4(7)$ \\
& Hypovascular & $4(7)$ \\
& Moderately vascular & $42(75)$ \\
& Highly vascular & $10(18)$ \\
& Absent & $40(71)$ \\
& Present & $16(29)$ \\
\hline \multirow{5}{*}{ Vascula } & & \\
& & \\
& &
\end{tabular}

Although approximately $61 \%$ of PAs in this series were oval in shape, their configuration varied highly ranging from nodular, fusiform, lobulated or crescent-like forms (fig 1) to a completely irregular outline. In terms of surface regularity, approximately $80 \%$ of PAs had a smooth contour. With regard to the internal echo texture, half of the PAs were homogeneous, while the other half exhibited internal components that distorted echo uniformity.

In terms of dominant internal echogenicity relative to that of thyroid gland, the PAs were overwhelmingly hypoechoic $(91 \%)$. Only one PA had a relatively hyperechoic appearance. In 10 PAs, a unique sonographic appearance of two concentric layers of different echogenicity was noted. The peripheral layer surrounded the central zone. Each of the layers covered more than $25 \%$ of the cross-sectional area of PAs. The mentioned dualconcentric echo pattern was in the form of an isoechoic central zone with a peripheral hypoechoic layer in 9 PAs (fig 2), and an isoechoic central part surrounded by a hyperechoic zone in $1 \mathrm{PA}$.

In 9 PAs (16\%), cystic changes were demonstrated in the lesion (fig 3). The PAs with cystic components were

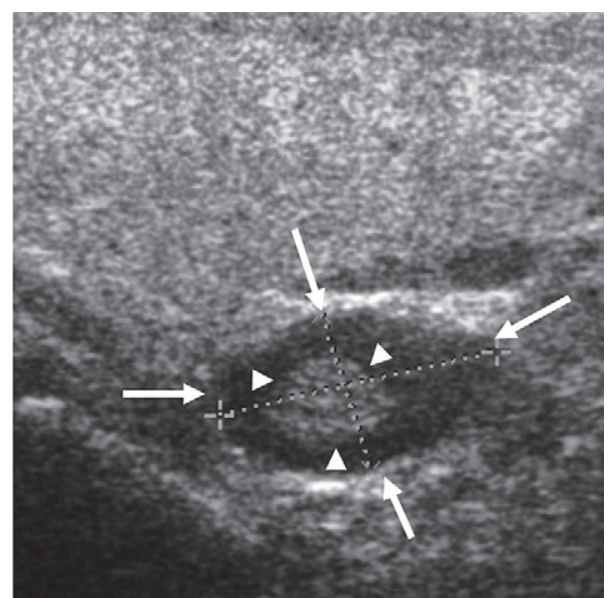

Fig 2. A parathyroid adenoma with dual-concentric echo pattern in a 47 year-old woman is shown in sagital ultrasound image. Note that contrary to a typical adenoma two distinct echogenic layers are present; isoechoic central zone (arrowheads) and peripheral hypoechoic layer (arrows) which may lead to misinterpretation with a lymph node.

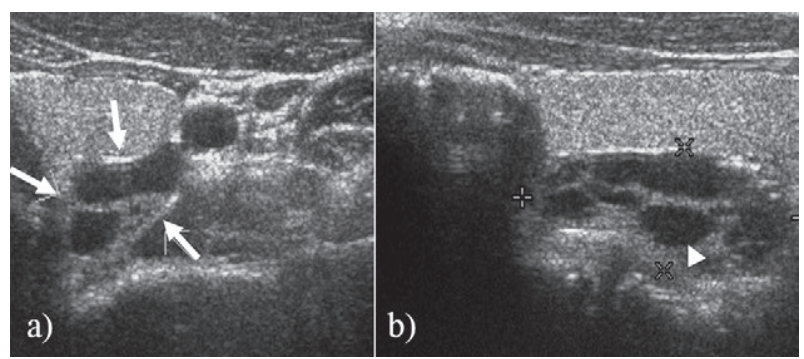

Fig 3. Transverse (a) and sagittal (b) ultrasound images in a 36 year-old woman. A parathyroid adenoma located close to the left lower portion of thyroid gland. Note that adenomatous tissue both comprises solid (arrows) and cystic areas (arrowhead).

significantly larger than the ones without (table II). In 4 PAs, these components were smaller than $5 \mathrm{~mm}$, while the remaining ones had larger cysts measuring up to $9 \mathrm{~mm}$ in long diameter. In addition to few punctate calcific foci of millimeter diameter that were demonstrated in 3 PAs, another PA was shown to have coarse calcification (fig 4).

Doppler sonographic evaluation of PAs revealed flow signals in all PAs in the series, demonstrating moderate to high degree of vascularity in $93 \%$ of them. Only 4 PAs were hypovascular, and had long diameters ranging from 1.3-2.8 cm, which overlapped the dimensions of remaning lesions. Nor was there any significant difference between hypovascular PAs and others in terms of lesion depth $(p=0.329)$. However, highly vascular PAs were significantly more superficially located compared to moderate-hypovascular PAs ( $\mathrm{p}=0.04)$. The "vascular arc" sign 
Table II. Comparison of the longest diameters of the PAs with regard to the presence or absence of the most common feature in the series for each US parameter.

\begin{tabular}{|c|c|c|c|}
\hline Sonographic parameter & $\begin{array}{l}\text { Longest diameter }(\mathrm{mm}) \text { of the PAs with } \\
\text { mostly demonstrated feature } \\
(\text { mean } \pm \text { SD) (n) }\end{array}$ & $\begin{array}{l}\text { Longest diameter }(\mathrm{mm}) \text { of the PAs } \\
\text { with other feature }(\mathrm{s}) \\
(\text { mean } \pm \text { SD) }(\mathrm{n})\end{array}$ & $p$ value \\
\hline Shape & $\begin{array}{l}\text { Oval } \\
1.7 \pm 0.8(34)\end{array}$ & $\begin{array}{l}\text { Others } \\
2.1 \pm 0.9(22)\end{array}$ & 0.129 \\
\hline Surface regularity & $\begin{array}{l}\text { Smooth } \\
1.9 \pm 0.9(45)\end{array}$ & $\begin{array}{l}\text { Irregular } \\
1.9 \pm 0.9(11)\end{array}$ & 0.838 \\
\hline Echo texture & $\begin{array}{l}\text { Homogeneous } \\
1.6 \pm 0.8(28)\end{array}$ & $\begin{array}{l}\text { Heterogeneous } \\
2.2 \pm 0.9(28)\end{array}$ & $0.030 \dagger$ \\
\hline Echogenicity (dominant) & $\begin{array}{l}\text { Hypoechoic: } \\
1.8 \pm 0.9(51)\end{array}$ & $\begin{array}{l}\text { Others: } \\
2.5 \pm 1.0(5)\end{array}$ & 0.208 \\
\hline Number of internal echo layers & $\begin{array}{l}\text { Single } \\
1.8 \pm 0.9(46)\end{array}$ & $\begin{array}{l}\text { Dual-concentric } \\
2.3 \pm 0.9(10)\end{array}$ & 0.139 \\
\hline Cystic component & $\begin{array}{l}\text { Cystic component absent } \\
1.6 \pm 0.7 \text { (47) }\end{array}$ & $\begin{array}{l}\text { Cystic component present } \\
2.3 \pm 0.7 \text { (9) }\end{array}$ & $0.014 \dagger$ \\
\hline Vascularity & $\begin{array}{l}\text { Moderately vascular } \\
1.9 \pm 0.9(42)\end{array}$ & $\begin{array}{l}\text { Highly vascular* } \\
2.1 \pm 0.9(10)\end{array}$ & 0.529 \\
\hline Vascular arc & $\begin{array}{l}\text { Negative } \\
1.8 \pm 0.9(40)\end{array}$ & $\begin{array}{l}\text { Positive } \\
2.0 \pm 0.9(16)\end{array}$ & 0.388 \\
\hline
\end{tabular}

(*) For statistical analysis, only highly vascular PAs were included. $(\dagger)$ Statistically significant difference.

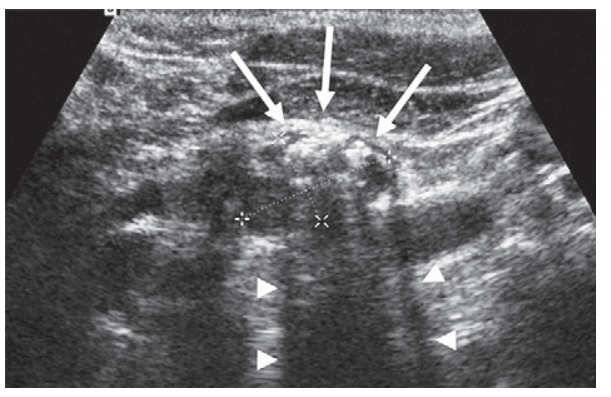

Fig 4. Transverse ultrasound image of a parathyroid adenoma with partially coarse calcification adjacent to the left lower pole of thyroid gland is depicted in a 29 year-old man. Hyperechoic calcifications (arrows) with strong acoustic shadowing (arrowheads) can be seen.

was present in 16 (29\%) of PAs (fig 5). These PAs did not demonstrate any significant difference in terms of lesion size or depth $(\mathrm{p}=0.348)$ relative to those PAs without "vascular arc" sign.

With regard to the different sonographic features of the PAs, mean lesion sizes in different subgroups were compared (tab II). PAs did not demonstrate any significant difference of longest diameter in terms of shape, surface regularity, echogenicity, presence of single or dual concentric echo layers, and degree of vascularity or presence/absence of vascular arc sign. However, heterogeneous PAs and PAs containing cystic components were significantly larger than homogeneous $(\mathrm{p}=0.03)$ and completely solid $(\mathrm{p}=0.01)$ lesions, respectively. There was no statistically significant relationship between serum calcium, intact parathormone and urinary calcium

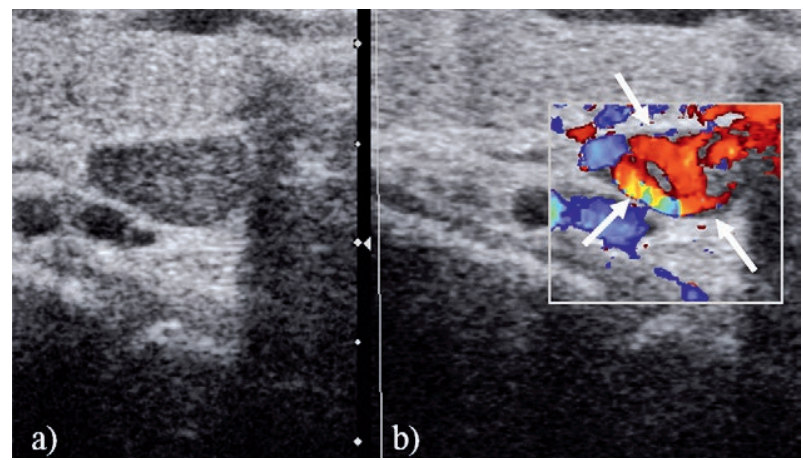

Fig 5. Sagittal grey scale (a) and Doppler ultrasound image (b) of a parathyroid adenoma measuring $1 \mathrm{~cm}$ in a 26 year-old woman is depicted. Note that an enlarged vessel wraps adenomatous tissue (arrows) giving the typical appearance of "vascular arc".

excretion levels with PAs showing different sonographic features (table III).

\section{Surgery:}

The time interval between the US examination and operation was between 0 and 309 days in the series (70 \pm 65 , mean $\pm \mathrm{SD}$ ). Eighty-one percent of the patients had been operated within 100 days after the US evaluation. All PAs were found surgically in the sites decribed with preoperative US. The locations of PAs were right superior, 2 (3.5\%); left superior, 7 (12.5\%); right inferior, 21 $(37.5 \%)$; left inferior, $24(42.8 \%)$. The remaining two $(3.5 \%)$ PAs were ectopic and found to be deeply located, adjacent to the bifurcation of brachiocephalic artery in the right half of the suprasternal fossa. The locations of PAs detected with US were in full compliance with surgical approach of adenomatous tissue. 
Table III. Statistical comparison of biochemical data with different ultrasonographic features using Mann-Whitney U test.

\begin{tabular}{|c|c|c|c|}
\hline & $\begin{array}{l}\text { Parathyroid hormone } \\
\text { levels } \\
\text { ( } p \text { value) }\end{array}$ & $\begin{array}{l}\text { Serum calcium } \\
\text { levels } \\
(p \text { value })\end{array}$ & $\begin{array}{l}\text { Urinary calcium ex- } \\
\text { cretion levels } \\
\text { ( } p \text { value) }\end{array}$ \\
\hline Shape (Oval vs. Others) & 0.162 & 0.301 & 0.269 \\
\hline Surface regularity (Smooth vs. Irregular) & 0.868 & 0.495 & 0.775 \\
\hline Echo texture (Homogeneous vs. Heterogeneous) & 0.257 & 0.181 & 0.328 \\
\hline Echogenicity (Hypoechoic vs. Others) & 0.924 & 0.320 & 0.845 \\
\hline $\begin{array}{l}\text { Number of internal echo layers } \\
\text { (Single vs. Dual-concentric) }\end{array}$ & 0.522 & 0.256 & 0.955 \\
\hline $\begin{array}{l}\text { Cystic component } \\
\text { (Absent vs. Present) }\end{array}$ & 0.214 & 0.091 & 0.271 \\
\hline $\begin{array}{l}\text { Vascularity } \\
\text { (Moderately vascular vs. Highly vascular*) }\end{array}$ & 0.250 & 0.771 & 0.350 \\
\hline Vascular arc (Negative vs. Positive) & 0.529 & 0.669 & 0.221 \\
\hline
\end{tabular}

(*) For statistical analysis, only highly vascular PAs were included; vs: Versus

\section{Histopathological results:}

Fifty-six PAs had a mean value of $1.73 \mathrm{~cm}( \pm 0.74 \mathrm{SD})$ ranging from 0.5 to $3.5 \mathrm{~cm}$ in the morphological evaluation. There was good agreement between the lengths of PAs acquired by sonograpical and histopathological methods (ICC 0.70; 95 CI values, 0.53-0.81; $\mathrm{p}<0.0001$ ). In 39 PAs (70\%) some normal parathyroid tissue around adenoma had been reported. No significant relationship between the presence of the normal parathyroid tissue around PA and any sonographic parameter could be demonstrated. Predominant cell types were chief cells in 33 PAs, oxyphilic cells in 6 PAs, water-clear cells in 8 PAs, and mixture of cells in 9 PAs.

The histopathological reevaluation of 54 PAs yielded ectatic vessels in $14(26 \%)$, central edema in $12(22 \%)$ and fibrous bands in 7 (13\%) of PAs. Blinded to the US observations, this analysis demonstrated significantly higher ratios of ectatic vessels $(67 \%$ vs. $18 \% ; p=0.02)$ and central edema ( $67 \%$ vs. $13 \% ; \mathrm{p}=<0.01)$ in the ten PAs with dual-concentric echo pattern compared to those without (fig 6). On the other hand, the PAs with the dual pattern did not demonstrate statistically significant dif-

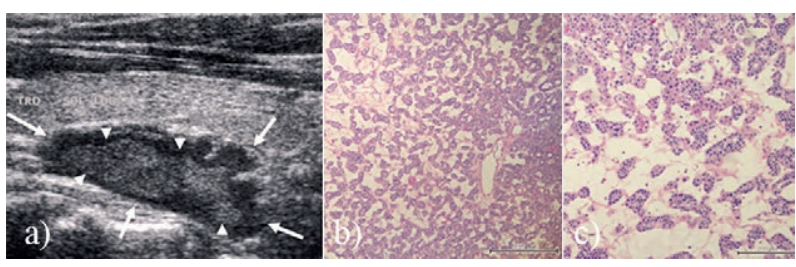

Fig 6. Sagittal ultrasound image (a) of a parathyroid adenoma with dual-concentric echo pattern in a 47 year-old woman. Isoechoic central zone (arrowheads) and peripheral hypoechoic layer (arrows) are seen in the ultrasound image. Low magnification (b) and high magnification view (c) of the same lesion from the central portion are depicted. Patients with this aforementioned novel ultrasound sign demonstrate more edema and ectatic vessels in the central portion with regard to those without this sign. ference in terms of predominant cell type, and presence of internal fibrous bands or normal parathyroid tissue around the lesion.

\section{Discussions}

In most cases there are four parathyroid glands located in the neck, but supernumerary and ectopic glands may occur and account for many cases of persistent or recurrent hyperparathyroidism after surgery [16]. Superior parathyroid glands together with the lateral lobes of thyroid gland orginate from the $4^{\text {th }}$ branchial pouch and migrate shorter distance compared to the inferior counterparts. On the other hand, the inferior parathyroids, developed from the third branchial pouch, migrate longer distance in embryologic descent. This is why ectopic adenomas orginate mostly from inferior parathyroids. Moreno et al reviewed the records of 271 patients operated on for sporadic primary hyperparathyroidism. According to this study the most prevalent location for adenomas was the inferior thyroid pole $26 \%$ [17]. Our study partially supports their findings. In our series we detected $80 \%$ of the PAs adjacent to the lower poles of thyroid gland. The second mostly encountered location was the left superior location. Regarding the limited number of cases in our series, larger scales of cases are needed to give a more reliable prevalence regarding the locations of PAs.

In typical cases PAs have uniformly hypoechoic appearance relative to the thyroid gland, and appear as well-circumscribed oval lesions. Compared to sestamibi scanning, US has the additional advantage of clearly demonstrating concomitant thyroid nodules which may cause false positive results with the scintigraphic technique. US may also reveal ectopic adenomas within the thyroid tissue, which are also hard to localize on sestamibi scanning [18-19]. The currently reached level of resolution capac- 
ity in modern US devices is far beyond those used in previous years; therefore, much more structural and hemodynamic details are obtainable in sonographic examinations today. That is why PAs without hypoechogenic appearance are not rare today, contrary to the older reports [21]. Chandramohan et al analyzed atypical US features of 26 parathyroid lesions. According to their study the ratio of non-hypoechoic PAs reaches up to $27 \%$ due to haemorrhage, hyalinisation and fibrosis in adenomatous tissue [22]. In the presented series, 9\% of the PAs were hyperor isoechoic relative to the echogenicity of thyroid gland.

During the growing phase, parathyroid adenomas assume the shape of space between longitudinally located tissues in the neck region which gives their characteristic elongated oval shape. If this process becomes more pronounced, an oval adenoma may become tubular or flat shaped. Due to asymmetric growth, cephalic and/or caudal end may become more circular. Additionally, atypical shapes such as triangular, conical, fusiform or lobulated can emerge in the growth phase of a PA [3]. Atypical shaped PAs with deeply hypoechoic internal echogenicity may be erroneously taken for regional veins in routine cervical scanning. In our series 5 PAs with fusiform or crescent-like shape had such configuration that presented some diagnostic challenge.

Instead of having single echogenicity, approximately $18 \%$ of the PAs in our series had dual, concentric echo structure constituted by areas of different echogenicity. The blinded histopathologic reevaluation of the PAs in the series demonstrated central edema and ectatic vessels more often compared to remaning PAs. Besides hemosiderin-laden macrophages and inflammation, edema may be seen in a PA, particularly in larger ones [23]. The sonographic lengths of PAs with dual, concentric echo structure were larger than those without, but the difference was not statistically significant. Although the limited number of cases with dual concentric echo pattern prevents full explanation, central edematous tissue and ectatic vessels may account for this unique US appearance, which had not been reported before, to the best of our knowledge.

Internal echo texture is one of the important diagnostic parameters that facilitate the diagnosis of PAs. Mostly being homogenous, PAs can be differentiated from regional lymph nodes, which are not rare especially in cases of chronic thyroiditis [24]. However, homogeneity of a PA is not a strict rule, especially when the US examination was performed with a high-resolution device. In our series, $50 \%$ of the PAs were visualized to display heterogenous texture. Apart from the high resolution technology of recent generation US machines, the dimensions of the PAs affect the sonographic internal appearance. It is known that PAs may develop heterogeneity of internal texture and cystic components, especially when they grow larger [19]. Microcysts may develop in parathyroid glands, possibly as a result of gradual local degeneration of the gland or retention of colloid secretion [25]. Cystic parathyroid adenomas may occur in approximately $2 \%$ of cases, and may be difficult to differentiate from the rare true parathyroid cysts [4]. The percentage of PAs with cystic component was $16 \%$ in our series. In accordance with these reports, the heterogenous PAs in our series were significantly larger than the homogeneous ones $(\mathrm{p}=0.03)$, as were the PAs with cystic elements when compared to those without ( $\mathrm{p}=0.01$ ).

Calcification is a rare sonographic finding in a PA [17]. In a recent study, Sidhu et al drew attention to the high positive predictive value of internal calcification in diagnosing parathyroid carcinoma [26]. Studying on adenomas of 15 $\mathrm{mm}$ or larger, they found calcified foci in 4 of 8 parathyroid carcinomas, while none of 61 benign lesions had any calcification. In our series, however, none of the operated parathyroid lesions with calcification was proved to be malignant. Therefore, we believe that larger and comparative studies are needed to reveal the real value of this sonographic finding in predicting malignancy of a parathyroid lesion

Regarding the fact that parathyroid adenomas are almost always hypervascular, color/power Doppler ultrasound is an indispensable tool in diagnosis [19]. There is always a large vessel in close proximity to the PA, usually contributing to the end artery which enters the adenoma from an anterior and superior position [9]. This large vessel creates the vascular arc sign in a typical adenoma [3]. However, this finding is not common in all parathyroid lesions. In our series 4 PAs (7\%) were hypovascular, and moreover we were able to detect the "vascular arc" appearance in only $29 \%$ of PAs. Contrary to our expectations, neither the hypovascular PAs nor the PAs without vascular arc sign were smaller or deeper in location than others in our series. This result is in favor of the assumption that the degree of vascularity in PAs is significantly influenced by individual characteristics of the lesions in addition to other technical parameters likely to diminish received Doppler signal. On the other hand, the analysis that yielded more superficial location of highly vascular lesions compared to others, underlines the effect of depth in the hypervascularity of PAs.

The retrospective study design and limited number of cases are the major limitations of this study. The ultrasonographic features of dual concentric echo sign could be better assessed if larger numbers of PAs with this aforementioned finding could be enrolled in the study.

\section{Conclusions}

Although most of the PAs present with typical features, some of them may exhibit variable sonographic 
appearances, dimensions and locations. Typical sonographic features such as ovoid shape, homogeneously hypoechoic pattern and vascular arc sign may not be present in all PAs. Without appropriate clinical guidance, proper knowledge of variations in US appearance, dedicated imaging technique and US devices of optimal quality, they may be easily overlooked. Except for homogeneously hypoechoic pattern and vascular arc sign which is conventionally seen in PAs, atypical features like dual concentric echo sign should alert radiologists for considering PA in differential diagnosis.

\section{Acknowledgement}

We thank Timur Kose from the Biostatistics Department of Ege University School of Medicine and Fatih Kayhan from Psychiatry Department of Mevlana University School of Medicine for their statistical advice and help during preparation of this article.

\section{Conflict of interest: none}

\section{References}

1. Lenchik L, Mitchell K. Hyperparathyroidism and renal osteodystrophy. In: El-Khoury G (eds.). Essentials of musculoskeletal imaging. Philadelphia PA, Churchill Livingstone 2003: 295-301.

2. Turgut B, Elagoz S, Erselcan T, et al. Preoperative localization of parathyroid adenomas with technetium-99m methoxyisobutylisonitrile imaging: relationship with P-glycoprotein expression, oxyphilic cell content, and tumoral tissue volume. Cancer Biother Radiopharm 2006; 21: 579-590.

3. Huppert BJ, Reading CC. The parathyroid glands. In: Rumack CM, Wilson SR, Charboneau JW, et al (eds.). Diagnostic Ultrasound 4th ed. Philadelphia (PA), Elsevier Mosby $2011: 750-71$.

4. Adler JT, Sippel RS, Chen H. The Influence of surgical approach on quality of life after parathyroid surgery. Ann Surg Oncol 2008; 15: 1559-1565.

5. Chen H, Sokoll LJ, Udelsman R. Outpatient minimally invasive parathyroidectomy: a combination of sestamibiSPECT localization, cervical block anesthesia, and intraoperative parathyroid hormone assay. Surgery 1999; 126: 1016-1022.

6. Khan AA, Clark OH. Handbook of parathyroid diseases: A Case-based Practical Guide. New York: Springer, 2012.

7. Abboud B, Sleilaty G, Rabaa L, et al. Ultrasonography: highly accuracy technique for preoperative localization of parathyroid adenoma. Laryngoscope 2008; 118: 1574-1578.

8. Baskin J. Durick D, Levine R. Thyroid ultrasound and ultrasound guided FNA. New York: Springer, 2008.

9. Randel SB, Gooding GA, Clark OH, Stein RM, Winkler B. Parathyroid variants: US evaluation. Radiology 1987; 165: 191-194.
10. Lane MJ, Desser TS, Weigel RJ, Jeffrey RB Jr. Use of color and power Doppler sonography to identifyfeeding arteries associated with parathyroid adenomas. Am J Roentgenol 1998; 171: 819-823.

11. Barraclough BM, Barraclough BH. Ultrasound of the thyroid and parathyroid glands. World J Surg 2000; 24: 158-165.

12. Mihai R, Barczynski M, Iacobone M, Sitges-Serra A. Surgical strategy for sporadic primary hyperparathyroidism an evidence-based approach to surgical strategy, patient selection, surgical access, and reoperations. Langenbecks Arch Surg 2009; 394: 785-798.

13. Miccoli P, Berti P, Materazzi G, Massi M, Picone A, Minuto MN. Results of video-assisted parathyroidectomy: single institution's six-year experience. World J Surg 2004; 28 : 1216-1218.

14. Di Stasio E, Carrozza C, Pio Lombardi C, et al. Parathyroidectomy monitored by intra-operative PTH: the relevance of the 20 min end-point. Clin Biochem 2007; 40: 595-603.

15. Carlson D. Parathyroid pathology: hyperparathyroidism and parathyroid tumors. Arch Pathol Lab Med 2010; 134: 1639-1644.

16. 16. Imachi H, Murao K, Kontani K, et al. Ectopic mediastinal parathyroid adenoma: a cause of acute pancreatitis. Endocrine 2009; 36: 194-197.

17. Moreno MA, Callender GG, Woodburn K, et al. Common locations of parathyroid adenomas. Ann Surg Oncol 2011; 18: 1047-1051.

18. Gilat H, Cohen M, Feinmesser R, et al. Minimally invasive procedure for resection of a parathyroid adenoma: the role of preoperative high-resolution ultrasonography. J Clin Ultrasound 2005; 33; 283-287.

19. Devcic Z, Jeffrey RB, Kamaya A, Desser TS. The elusive parathyroid adenoma: techniques for detection. Ultrasound Q 2013; 29: 179-187.

20. Rubello D, Giannini S, Martini C, et al. Minimally invasive radio-guided parathyroidectomy. Biomed Pharmacother 2006; 60: 134-138.

21. Pesenti M, Frasoldati A, Azzarito C, Valcavi R. Parathyroid incidentaloma discovered during thyroid ultrasound imaging. J Endocrinol Invest 1999; 22; 796-799.

22. Chandramohan A, Sathyakumar K, John RA, et al. Atypical ultrasound features of parathyroid tumours may bear a relationship to their clinical and biochemical presentation. Insights Imaging 2014; 5: 103-111.

23. Lester DR. Thompson. Head and Neck Pathology. Churchill Livingstone: Elsevier, 2013.

24. 24. Ozdemir D, Arpaci D, Ucler R, Cuhaci N, Ersoy R, Cakir B. Parathyroid incidentalomas detected during thyroid ultrasonography and effect of chronic thyroiditis on false positive parathyroid lesions. Endocrine 2012; 42: 616-621.

25. Ippolito G, Palazzo FF, Sebag F, Sierra M, De Micco C, Henry JF. A single-institution 25-year review of true parathyroid cysts. Langenbecks Arch Surg 2006; 391:13-18.

26. Sidhu PS, Talat N, Patel P, Mulholland NJ, Schulte KM. UItrasound features of malignancy in the preoperative diagnosis of parathyroid cancer: a retrospective analysis of parathyroid tumours larger than 15mm. Eur Radiol 2011; 21: 1865-1873. 